RESEARCH ARTICLE

\title{
Liquid crystal behaviour of three novel glycosides
}

\author{
A.R.N.M. Abeyrathne ${ }^{1}$, A.D.L. Chandani Perera ${ }^{2}$ and D.N. Karunaratne ${ }^{2^{*}}$ \\ ${ }^{1}$ Postgraduate Institute of Science, University of Peradeniya, Peradeniya. \\ ${ }^{2}$ Department of Chemistry, Faculty of Science, University of Peradeniya, Peradeniya.
}

Revised: 03 November 2011 ; Accepted: 18 November 2011

\begin{abstract}
Novel glycosides synthesized by linking D-glucose to three different non-polar aglycones, cinnamyl alcohol, chloroxylenol and 3-pentadecylphenol showed both thermotropic and lyotropic liquid crystal phases. The effect of inclusion of a rigid spacer in between hydrophilic and hydrophobic parts on the stability of liquid crystal phases is investigated. Both acetylated and deacetylated compounds exhibited thermotropic and lyotropic liquid crystal behaviour. The liquid crystal phases were confirmed as smectic A, smectic $\mathrm{B}$ and hexagonal columnar by X-ray studies.
\end{abstract}

Keywords: Glycolipids, liquid crystal, lyotropic, thermotropic.

\section{INTRODUCTION}

Studies conducted on carbohydrates with alkyl or aryl substituents have shown liquid crystalline properties with clearly defined thermotropic and lyotropic mesophase behaviour. These carbohydrate derived liquid crystals have technical and biological applications in a wide spectrum i.e. thermotropic and lyotropic liquid crystals, surfactants, lubricants, drug delivery, solubilization and crystallization of membrane enzymes etc. (Vill \& Hashim, 2002).

Liquid crystal state is a distinct state of matter observed between the crystalline solid, and isotropic liquid (Vill, 1998). The presence of liquid crystallinity in glycolipids mainly depends on the type of carbohydrate used as the hydrophilic part, the hydrophobic part linked to the carbohydrate, the presence of a rigid spacer in between the polar and non-polar parts such as a phenyl group and the type of linkage like ether or ester. The overall molecule itself gives rise to the mesophase behaviour (Smiths et al., 1997).
Thermotropic liquid crystal formation of carbohydrate derived liquid crystals is a two-stage melting process showing two different melting points (melting point and clearing point), because carbohydrates form hard crystals whereas hydrocarbons form soft crystals giving high and low melting points, respectively. Upon heating the glycolipid, at the melting point, first the hydrocarbons start to melt losing the three dimensional structure while at the clearing point, the hard carbohydrate part starts to melt and forms the isotropic liquid at a higher temperature. In-between these two melting points the liquid crystal mesophases appear to be stable. Normally, thermotropic mesophase behaviour includes several phase transitions such as crystal to crystal, crystal to mesophase, and mesophase to mesophase. For carbohydrate derived liquid crystals, crystal to crystal transition can be observed before the melting point. Most of the carbohydrate liquid crystals show smectic phases. However, cholestric phase is rarely observed, although these compounds have chirality in the sugar part (Jeffrey, 1986).

The aim of the present research was to synthesize novel carbohydrate derived liquid crystals and to study the effect of alkyl and aryl stability of liquid crystal phases upon inclusion of a rigid spacer in between hydrophilic and hydrophobic parts. In this work, glucose was used as the source material and three novel glycosides; cinnamyl 2, 3, 4, 6-tetra-O-acetyl- $\beta$-D-glucopyranoside (1a), 4-chloro-3, 5-dimethylphenyl 2, 3, 4, 6-tetra-O-acetyl$\beta$-D-glucopyranoside (1b) and 3-pentadecylphenyl 2, 3, 4, 6-tetra-O-acetyl-1-O- $\beta$-D-glucopyranoside (1c), were synthesized (Table 1) by making simple modifications to previously reported procedures (Smiths et al., 1996, 1997). Further they were fully characterized using

\footnotetext{
*Corresponding author (nedrak@pdn.ac.1k)
} 
Nuclear Magnetic Resonance spectroscopy (NMR), Fourier Transform Infrared spectroscopy (FTIR) and Mass Spectroscopy (MS). The thermotropic liquid crystal behaviour was studied using optical polarizing microscope and the phase transitions were observed by thermo-microscopy and further analyzed by Differential Scanning Calorimetry (DSC). The layered structure and the confirmation of the mesophases were done using $\mathrm{X}$-ray diffractometric studies (XRD). Furthermore, the thermotropic and lyotropic behaviour of the deacetylated products of the above three compounds were also studied.

\section{METHODS AND MATERIALS}

\section{Instrumentation}

Melting points were determined with a melting point apparatus (SMP1, Stuart Scientific, UK) and the TLC was performed on silica gel 60F- 254 (Merck) and visualized with UV light and anisaldehyde spray reagent. The chemical structures of all products were confirmed by spectroscopic techniques; NMR spectroscopy (300 $\mathrm{MHz}$ Varian VTR-300 spectrometer); chemical shifts are relative to $\mathrm{CDCl}_{3}$, infrared spectra were recorded on a FTIR spectrometer (IR Prestige- 21, SHIMADZU) and mass spectra were obtained using GC-MS spectrometer (Varian 2000, Saturn GC/ MS/ MS).

Identification of optical texture was carried out using an optical polarizing microscope (Leica-DMLS, USA) and optical micrographs were taken under crossed polarizers. Quantitative thermal analysis (melting point; $\mathrm{Mp}$, clearing point; cp) was performed using a Perkin Elmer PC Series DSC 7 with a heating/cooling rate of $2{ }^{\circ} \mathrm{C} \mathrm{min}^{-1}$. Thermo-microscopy was performed using the polarizing microscope, a hot stage and a temperature control unit (Model SU, Chino; Eurotherm 2116). X-ray analysis was done using the X-ray diffractometer D-5000 (Siemens, Germany); $2 \theta$ range from $2.0^{\circ}$ to $20.0^{\circ}$ at $1.5406 \mathrm{~A}^{\circ}$ wavelength with a scan rate of $0.0001^{\circ} / \mathrm{min}$ and step size of $7 \mathrm{~S}$. The compounds were heated to isotropic phase and cooled down to room temperature prior to recording the diffraction pattern at room temperature.

\section{Synthesis of glucosides}

The general procedures (Smiths et al., 1996,1997; Mattsson et al., 2007) used for the synthesis of compounds are described below. All the reagents and solvents were purchased from Sigma Chemicals (USA) and some

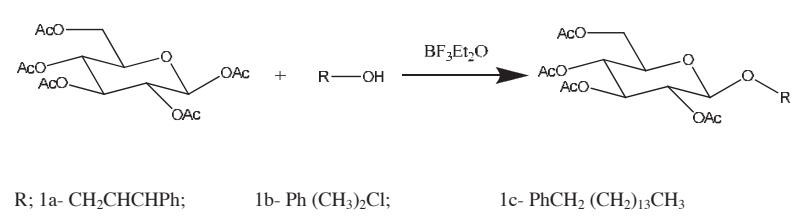

Figure 1: Synthetic route for glucosides

were purified before use. The synthetic route used in the present work is given in Figure 1.

a) Synthesis of cinnamyl 2, 3, 4, 6-tetra- $\underline{0}$-acetyl- $\beta$ D-glucopyranoside (1a): Boron trifluoride etherate (14 mmol) was added to a stirred solution of penta-Oacetyl glucopyranoside (PAGP) (10 mmol) and cinnamyl alcohol $(14 \mathrm{mmol})$ in dry $\mathrm{CH}_{2} \mathrm{Cl}_{2}(100 \mathrm{~mL})$ under nitrogen atmosphere, via a syringe, stirred for $6 \mathrm{~h}$ at room temperature and quenched with saturated hydrogen carbonate solution. The organic layer was separated and washed successively with aqueous $\mathrm{NaHCO}_{3}$ and (once) with water, dried over $\mathrm{MgSO}_{4}$, and concentrated. The extract was then purified by column chromatography using petroleum ether and ethyl acetate as the solvent system to get the desired product.

Yield; $67 \%$. Mass; m/z \{ $\mathrm{M}^{+}(\mathrm{cal}=464), 417,331$, 326, 283, 271, 211, 169, 134, 127, 115 \}. FTIR; $v_{\max }=$ $1745(\mathrm{C}=\mathrm{O}), 1516\left(\mathrm{CH}_{3}\right), 1550(\mathrm{C}=\mathrm{C}), 1000-1300$ (C-O), 1402.02 ( arom H), 600- 1500 (sugar part). ${ }^{1} \mathrm{H} ; \delta$ $=2.09,2.10,2.15,2.24(4 \mathrm{~s}, 12 \mathrm{H}, 4 \times$ acetyl $), 4.22(\mathrm{~m}$, $1 \mathrm{H}, \mathrm{H}-5), 4.15$ (d, 1 H, H-6), 6.38 (d, J, 1-H, H-1), 5.15, 5.15, 5.43 (3 dd, $3 \mathrm{H}, \mathrm{H}-2 / 4), 7.20-7.40$ (m, $5 \mathrm{H}),(\mathrm{d}$, $\left.2 \mathrm{H}, \mathrm{H}-1^{\prime}\right),\left(\mathrm{m}, 1 \mathrm{H}, \mathrm{H}-2^{\prime}\right), 6.40$ (d, $\left.1 \mathrm{H}, \mathrm{H}-3^{\prime}\right) .{ }^{13} \mathrm{C} ; \delta=$ 20.25, 20.35, 20.58, 20.95 (q, $4 \times \mathrm{CH}_{3}$ acetyl), 61.58 (t, C-6), 69.69, 69.85, 70.26, 70.85 (4 d, C-2/5), 89.31 (d, C-1), 67.66 (t, C-1'), 127.71 (d, C-2'), 128.64 (d, C-3'), 129.58 (s, arom C), $126.21-126.63$ (5 d, arom CH), 168.83, 169.77, 170.31, 170.71 (4 s, CO acetyl). Mp; 75 $-83^{\circ} \mathrm{C}$, cp: $83^{\circ} \mathrm{C}$, cooling point; $62-55^{\circ} \mathrm{C}$.

b) Synthesis of 4-chloro-3, 5-dimethylphenyl 2, 3, 4, 6-tetra-O-acetyl- $\beta$-D-glucopyranoside (1b): PAGP (1.95 g, $5 \mathrm{mmol}$ ) and 4-chloro-3,5-dimethylphenol $(0.7831 \mathrm{~g}, 5 \mathrm{mmol})$ were dissolved in anhydrous $\mathrm{CH}_{2} \mathrm{Cl}_{2}$ $(10 \mathrm{~mL})$, followed by the addition of boron trifluoride etherate $(0.625 \mathrm{~mL}, 5 \mathrm{mmol})$ and stirred for $24 \mathrm{~h}$ at room temperature. The mixture was poured into $5 \%$ aqueous $\mathrm{NaHCO}_{3}(20 \mathrm{~mL})$ and the organic layer was separated, washed with aqueous $\mathrm{NaHCO}_{3}$ and once with water, dried over $\mathrm{Na}_{2} \mathrm{SO}_{4}$ and concentrated. The crude product was recrystallized from ethanol to yield the pure product. 
Yield; 81\%. Mass; $\mathrm{m} / \mathrm{z}\left\{\mathrm{M}^{+}(\mathrm{cal}=486), \mathrm{M}^{+}+1=487\right.$, 332, 272, 211, 156\}. FTIR; $v_{\max }=1739(\mathrm{C}=\mathrm{O}), 1516$ $\left(\mathrm{CH}_{3}\right), 1429(\mathrm{C}=\mathrm{C}), 1000-1300(\mathrm{C}-\mathrm{O}), 1402.02(\operatorname{arom~H})$, 600- 1500 (sugar part). ${ }^{1} \mathrm{H} ; \delta=2.03-2.09$ (4 s, $12 \mathrm{H}, 4 \times$ acetyl), 4.22 (m, $1 \mathrm{H}, \mathrm{H}-5), 4.13$ (d, $1 \mathrm{H}, \mathrm{H}-6), 6.34$ (d, J, 1-H, H-1), 5.14, 5.08, 5.47 (3 dd, 3 H, H-2, 3, 4), 6.57 (s, $2 \mathrm{H}$, arom H), 2.31 (s, $6 \mathrm{H}, 2 \times$ methyl). ${ }^{13} \mathrm{C} ; \delta=20.89(\mathrm{q}$, $4 \times \mathrm{CH}_{3}$ acetyl), 61.78 (t, C-6), 69.50, 70.13, 70.04, 68.18 (4 d, C-2, 3, 4, 5), 89.33 (d, C-1), 115.52 (d, $2 \times$ arom $\mathrm{CH}), 127.70(\mathrm{~s}, \operatorname{arom} \mathrm{C}), 137.55\left(\mathrm{~s}, 2 \times\right.$ arom $\left.\mathrm{C}-\mathrm{CH}_{3}\right)$, 153.78 (s, arom C-O), $126.21-126.63$ (5 d, arom CH), 169.16, 169.81, 170.07, 170.64 (4 s, CO acetyl). Mp; 54 - $69{ }^{\circ} \mathrm{C}$, cp: $95-98^{\circ} \mathrm{C}$, cooling point; $64-83{ }^{\circ} \mathrm{C}$.

c) Synthesis of tetra-O-acetyl- $\beta$-glucopyranosyl chloride (GPTACI): PAGP (1g) and crushed anhydrous aluminium chloride (0.36 g) were added to pure, dry $\mathrm{CHCl}_{3}$ and shaken for $1.5 \mathrm{~h}$ at room temperature. The precipitate was washed with benzene $(1.25 \mathrm{~mL})$ and the filtrate was added to $0{ }^{\circ} \mathrm{C}$ water $(2.5 \mathrm{~mL})$ in a separatory funnel and shaken well. The organic layer was filtered via a filter paper wetted with dry benzene and immediately evaporated under reduced pressure to a syrupy residue. It was dissolved in a mixture of anhydrous ether $(2.5 \mathrm{~mL})$ and dry petroleum ether $(1.25 \mathrm{~mL})$. The crystals formed were filtered, washed with petroleum ether and dried under reduced pressure over phosphorous pentoxide and sodium hydroxide pellets. Finally the crude product was recrystallized using a 1:1 mixture of ether and petroleum ether.

Yield ${ }^{1} \mathrm{H} ; \delta=2.07,2.08,2.09,2.10,2.15(5 \mathrm{~s}, 15 \mathrm{H}$, $5 \times$ acetyl), $4.13(\mathrm{~m}, 1 \mathrm{H}, \mathrm{H}-5), 4.20(\mathrm{~d}, 1 \mathrm{H}, \mathrm{H}-6), 6.35$ (d, J, 1-H, H-1), 5.13, 5.15, 5.44 (3 dd, 3 H, H-2, -3 and -4). ${ }^{13} \mathrm{C} ; \delta=168.63,169.30,169.55,170.10,170.45$ (q, $5 \times \mathrm{CO}$ acetyl), 19.56, 20.12, 20.25, 20.35, 20.57, (q, 5 $\times \mathrm{CH}_{3}$ acetyl), $60.40(\mathrm{t}, \mathrm{C}-6), 68.55,68.90,69.16,69.68$ (4 d, C-2/5), 87.90 (d, C-1). Mp; $105-115^{\circ} \mathrm{C}$.

d) Synthesis of 3- pentadecylphenyl 2, 3, 4, 6-tetraO-acetyl-1-O- $\boldsymbol{\beta}$-D-glucopyranoside (1c): To a stirred solution of GPTACl $(10 \mathrm{mmol})$ and 3-pentadecylphenol (14 mmol) in dry dichloromethane $(100 \mathrm{~mL})$, boron trifluoride etherate $(14 \mathrm{mmol})$ was added via a syringe. The solution was stirred for $48 \mathrm{~h}$ at room temperature after which it was quenched with saturated hydrogen carbonate solution and the organic layer was separated. The aqueous phase was extracted twice with dichloromethane and the combined organic extracts were washed with water and dried over magnesium sulphate. It was filtered and evaporated to obtain the crude extract. The extract was then purified by column chromatography using hexane: dichloromethane $(1: 1)$ as the solvent system and then with acetone $(100 \%)$ to give the desired product.
Yield: $67 \%$ : Mass; m/z $\left\{\mathrm{M}^{+}(\mathrm{cal}=634), 331,229,186\right.$, 211, 93, 77. FTIR; vmax $=2852-2924\left(\left(\mathrm{CH}_{2}\right)_{14} \mathrm{CH}_{3}\right), 1751$ $(\mathrm{C}=\mathrm{O}), 1166$ (C-O-R), 1585-1616( arom H), 600- 1500 (sugar part). ${ }^{1} \mathrm{H} ; \delta=2.02,2.04,2.05,2.06(4 \mathrm{~s}, 12 \mathrm{H}, 4$ $\times$ acetyl), $5.04(\mathrm{~m}, 1 \mathrm{H}, \mathrm{H}-5), 3.80(\mathrm{~d}, 1 \mathrm{H}, \mathrm{H}-6), 6.32$ (d, 1-H, H-1), 5.28, 5.70, 4.28 (3 dd, 3 H, H-2, 3, 4), 6.78- 7.20 (s, d, dd, d, $4 \mathrm{H}$, arom H-2, 4, 5, 6), 2.56 (t, $2 \mathrm{H}$, alkane chain-7), 1.58 ( $\mathrm{m}, 2 \mathrm{H}$, alkane chain-8), 1.25 (m, $18 \mathrm{H}$, alkane chain-9-17), 1.30 (m, $2 \mathrm{H}$, alkane chain- 20), 0.85 (d, $3 \mathrm{H}$, alkane chain-21). ${ }^{13} \mathrm{C} ; \delta 20.62$ (q, $4 \times \mathrm{CH}_{3}$ acetyl), 61.89 (t, C-6), 71.50, 70.06, 70.39, 73.01(4 d, C-2, 3, 4, 5), 99.36 (d, C-1), 157.13 (s, arom C-1), 123.38, 123.68(d, arom C-2,5), 116.91(d, arom C-4), 113.78(s, arom C-3), 129.54(d, arom C-6), 36.14(t, alkane C-7), 31.52(t, alkane C-8), 29.71(t, alkane C-9,18), 29.89(t, alkane C-10,17), 32.12(t, alkane C-19), 22.88(t, alkane C-20), 14.30(q, alkane C-21). Mp; 45-51 ${ }^{\circ} \mathrm{C}$, cp: 63-64 ${ }^{\circ} \mathrm{C}$, cooling point; $54-58{ }^{\circ} \mathrm{C}$.

e) Deacetylation of compounds, 1a, 1b and 1c: Acetylated glucoside (1 eq), triethyl amine (3 eq) and lithium bromide (10 eq) were stirred in a solution of acetonitrile $(1 \mathrm{~mL})$ and distilled water $(2 \% \mathrm{vol})$, at room temperature for $24 \mathrm{~h}$. It was evaporated under reduced pressure and filtered to obtain the crude product followed by recystallization with the solvent system of petroleum ether: ethyl acetate $(4: 1)$.

Table 1: Chemical structures of the acetylated and deacetylated glucosides

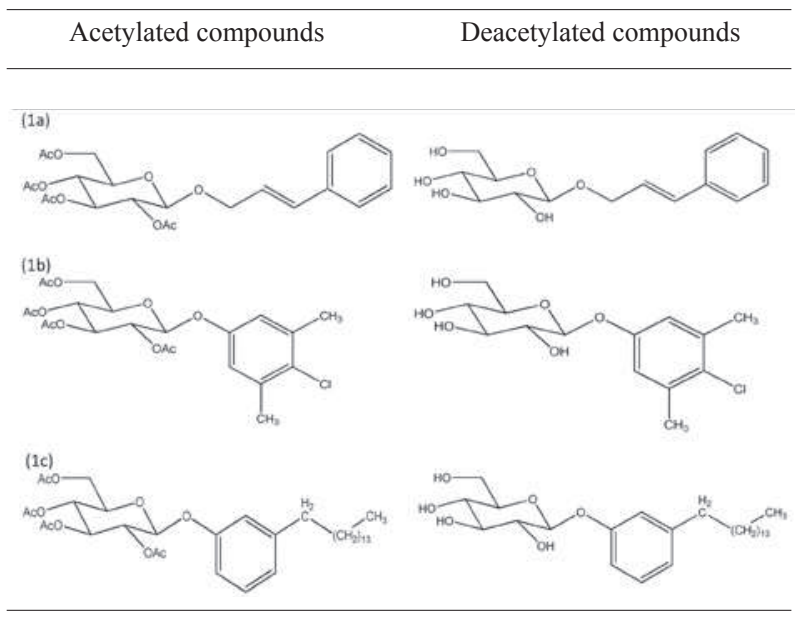

\section{RESULTS AND DISCUSSION}

In the synthetic scheme of alkyl glycoside (1a), the formation of the glycosidic bond is done using a previously reported method (Smiths et al., 1996;1997) 
with boron trifluoride etherate $\left(\mathrm{BF}_{3} \mathrm{Et}_{2} \mathrm{O}\right)$ as the catalyst in a nitrogen environment for 6 hours and the final product was obtained in high yield. But the synthesis of aryl glycosides ( $1 \mathrm{~b}$ and $1 \mathrm{c})$ was found to be difficult using the same method. Thus, simple modifications had been done. For the synthesis of $1 \mathrm{~b}$, equimolar amounts of PAGP, chloroxylenol and $\mathrm{BF}_{3} \mathrm{Et}_{2} \mathrm{O}$ were mixed and stirred for 24 hours at room temperature. For compound $1 \mathrm{c}$, although the same method was used as in the previous case, the desired product was not obtained. This could be due to the poor acceptor nature of the phenol derivative, since the pentadodecyl group can increase the electron density of the ring. Therefore, glucosyl donor was activated by converting PAGP into PAG chloride and reacted with the alcohol for 48 hours until the desired product was obtained in high yield. The $\beta$-configuration of purified aryl glucosides were established by both ${ }^{1} \mathrm{H}$ and ${ }^{13} \mathrm{C}$ NMR. The deacetylation of these compounds was tried using several possible methods. But it was not achieved in good yield because of the use of strong bases, which cause the breakage of the glycosidic linkage between the anomeric carbon and the aryl group. The deacetylation, in the presence of lithium bromide and triethyl amine (FodorCsorba et al., 2006) was successful, although the yield was much lower. Both the acetylated and deacetylated glucosides of 1a were light yellow colour amorphous compounds while those of compound $1 \mathrm{~b}$ were white amorphous solids. The acetylated compound, 1c, was off-white colour amorphous whereas the deacetylated product of $1 \mathrm{c}$ was a white jelly-like compound.

Thermotropic liquid crystal phases of all three compounds were observed by thermo-microscopy. The liquid crystal mesophases are more stabilized by the inclusion of a rigid spacer unit, such as a phenyl group in between the sugar part and the nonpolar part as a linking group. Phase transition temperatures observed for acetylated compounds using thermo-microscopy and DSC are compared in Table 2.
The phase transition temperatures given in Table 2 were measured by DSC in the first heating and cooling cycles. The DSC thermograms obtained for the compounds gave somewhat similar melting and clearing points to those obtained from thermo-microscopy. Also one crystal-to-crystal transition temperature was observed in each thermogram at low temperature, which is significant in the DSC thermograms of carbohydrate liquid crystals. Upon heating, compound 1a started melting around $75^{\circ} \mathrm{C}$ and became completely isotropic around $83{ }^{\circ} \mathrm{C}$. On the other hand, upon cooling, a fan/ focal conical like texture was observed (Figure 2a), which is common for both smectic A and hexagonal columnar mesophases. In this texture the two pairs of brushes do not contact at the center of the fan like domain. According to the XRD data, compound 1a showed a single, intense peak in the small angle region, at $2 \theta=2.30^{\circ}$ with a d-spacing of $38.37 \mathrm{~A}^{\circ}$ (Figure $3 \mathrm{a}$ ), which is characteristic for smectic phases. For columnar hexagonal phases, three reflection peaks (one intense peak and two smaller peaks) should be observed in the small angle region. Thus, it can be concluded that compound 1a exhibits smectic A liquid crystal phase. The molecular length of the compound, obtained from Gaussian modelling, a simple molecular modelling package, was around $14.38 \mathrm{~A}^{\circ}$. Thus the $\mathrm{d}$-spacing is 2.7 times the molecular length and it seems that the molecules may exist in a dimer form with a headto-head bilayer molecular packing in the mesophase (Jeffrey, 1986; Wingert et al., 1995). In this compound the hydrophilic sugar part and lipophilic cinnamyl part are comparable in size and would satisfy lipophilic and hydrophilic balance to form smectic A phase.

The inclusion of a rigid spacer unit, such as a phenyl group in between the sugar part and the nonpolar part as a linking group lowered the clearing point. Comparison of compound 1a with 1c indicates a long alkyl chain linked to the phenyl group in compound $1 \mathrm{c}$, resulting in lower melting and clearing points compared to the

Table 2: Quantitative thermal analysis of acetylated glucosides

\begin{tabular}{|c|c|c|c|c|c|c|c|}
\hline \multirow[t]{4}{*}{ Compound } & \multicolumn{7}{|c|}{ Method } \\
\hline & \multicolumn{4}{|c|}{ DSC } & \multicolumn{3}{|c|}{ Thermo-microscopy } \\
\hline & & Heating & & Cooling & & & Cooling \\
\hline & T.pt ${ }^{\mathrm{a} /}{ }^{\circ} \mathrm{C}$ & $\mathrm{Mp}^{\mathrm{b}} /{ }^{\circ} \mathrm{C}$ & $\mathrm{cp}^{\mathrm{c} /}{ }^{\circ} \mathrm{C}$ & T.pt $/{ }^{\circ} \mathrm{C}$ & $\mathrm{Mp} /{ }^{\circ} \mathrm{C}$ & $\mathrm{cp} /{ }^{\circ} \mathrm{C}$ & T.pt $/{ }^{\circ} \mathrm{C}$ \\
\hline $1 \mathrm{a}$ & $41,62,79$ & 62 & 97 & 53 & 75 & 83 & $62-55$ \\
\hline $1 b$ & 37,83 & 54 & 98 & $37,46,74$ & 69 & 95 & $64-73$ \\
\hline $1 \mathrm{c}$ & 36,57 & 51 & 64 & 48,54 & 45 & 63 & 58 \\
\hline
\end{tabular}

${ }^{a}$ T.pt; phase transition temperatures, ${ }^{b}$ m.p; melting point, ${ }^{c}$ c.p; clearing point 


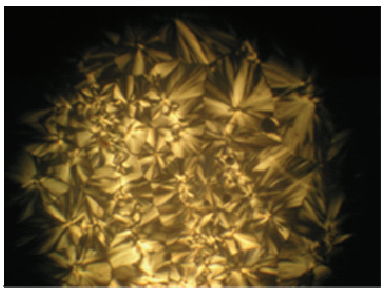

(a)

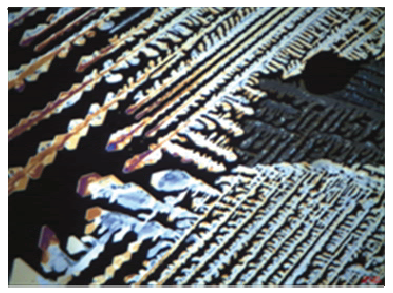

(c)

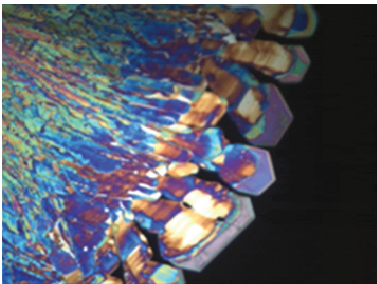

(b)

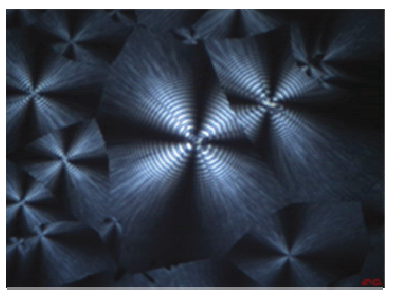

(d)
Figure 2: (a) Focal conical texture of compound $1 \mathrm{a}\left(55-62^{\circ} \mathrm{C}, 400 \times\right)$, (b) mosaic texture of compound $1 \mathrm{~b}\left(64-73{ }^{\circ} \mathrm{C}, 400 \times\right)$, (c) dendritic growth of mosaic texture of compound $1 \mathrm{~b}$ and $(\mathrm{d})$ focal conical texture of compound $1 \mathrm{c}\left(58^{\circ} \mathrm{C}, 400 \times\right)$ taken under crossed polarizers.

other two compounds. Generally, the longer the nonpolar part, the more smectic or columnar phases are observed. In accordance with that, compound 1c gave perfect focal conical texture under the crossed polarizer and analyzer around $58{ }^{\circ} \mathrm{C}$ (Figure 2d). However, this compound also showed typical carbohydrate mesogenic property with a crystal-to-crystal transition at $37{ }^{\circ} \mathrm{C}$ in the DSC thermogram before the liquid crystal transition. The detected XRD reflection peak at $2 \theta=2.61^{\circ}$ in the small angle region (Figure $3 \mathrm{~b}$ ) corresponding to $\mathrm{d}$-spacing of $33.03 \mathrm{~A}^{\circ}$ in the observed texture is almost similar to the molecular length, $29.58 \mathrm{~A}^{\circ}$ at its most stable conformation. Accordingly, molecules may exist in monolayer molecular packing (Jeffrey, 1986; Wingert et al., 1995). Smaller peaks in addition to the most distinct peak, in the low angle region confirm the liquid crystal phase of this compound as the hexagonal columnar phase. Further, the diffuse peak in the wide-angle region suggests the fluidity due to the long alkyl chain.

For compound $1 \mathrm{~b}$, melting and clearing points were observed around $69^{\circ} \mathrm{C}$ and $95^{\circ} \mathrm{C}$ and the DSC heating scan further evidenced this. Also one endothermic peak was observed around $37^{\circ} \mathrm{C}$, which may be due to the crystal-to-crystal transition. Upon cooling a mosaic texture was observed (Figure 2b) around $64-73{ }^{\circ} \mathrm{C}$ and in the thermogram an endothermic peak related to this liquid crystal phase formation was observed around $74{ }^{\circ} \mathrm{C}$.

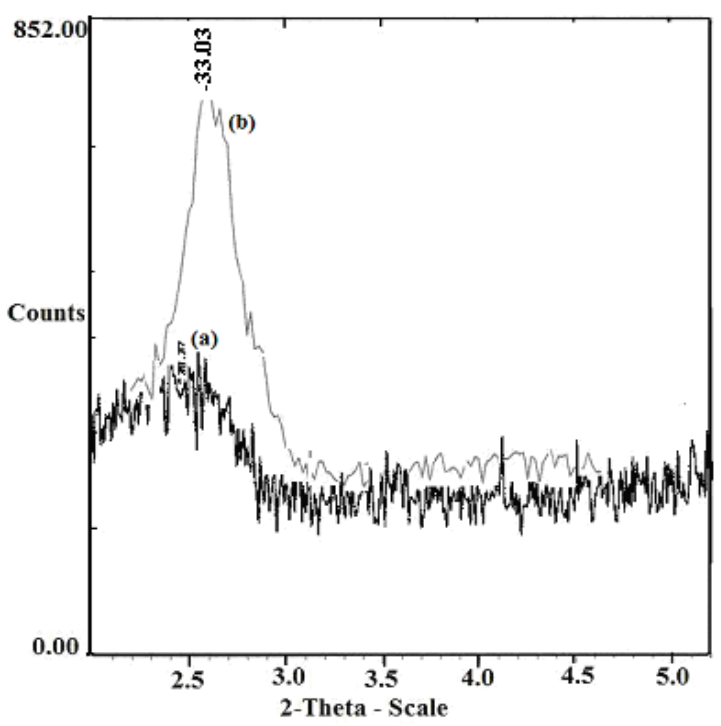

Figure 3: Small angle region of XRD pattern of (a) compound 1a and (b) compound $1 \mathrm{c}$ taken at $25^{\circ} \mathrm{C}$

Dendritic growth of mosaic textures (Figure 2c), which is significant in smectic B phases was also observed for compound $1 \mathrm{~b}$. Hence, there is a possibility that this mesophase is a columnar $\mathrm{B}_{1}$ phase (Smiths, 1996). In the $\mathrm{X}$-ray diffractogram, a peak in the small angle region $\left(2 \theta=4.24^{\circ}\right)$ was observed with a d-spacing of $20.81 \mathrm{~A}^{\circ}$ suggesting a layered structure. Also the d-spacing value is greater than 1 and less than 2 times the molecular length, $12.81 \mathrm{~A}^{\circ}$. Accordingly, molecules may exist in bilayers forming a head-to-head molecular packing in two dimensions. The additional second peak at wide angle region $\left(2 \theta=9.36^{\circ}\right)$ with a d-spacing of $9.44 \mathrm{~A}^{\circ}$ is a diffuse peak, which shows fluidity in a plane structure (Jeffrey, 1986; Wingert et al., 1995; Fodor-Csorba et al., 2006). This compound has only a phenyl ring and a sugar moiety and hence the molecule is not flexible enough to form smectic phase.

Further, the three compounds discussed above are amphotropic liquid crystals, because they show both thermotropic and lyotropic properties. The lyotropic behaviour is very similar to thermotropic behaviour, except the fact that lyotropic phase behaviour changes with both the amount of solvent and temperature. Thus, a compound may have one thermotropic phase and several lyotropic phases (Vill \& Hashim, 2002). The observed lyotropic textures with dichloromethane, a nonpolar solvent, under the polarizing microscope are given in Figure 4. 
The deacetylated products of the above compounds also showed both thermotropic and lyotropic properties (Figure 5). The melting and clearing points observed for all the deacetylated compounds by thermo-microscopy are given in the Table 3. Although the melting points of both acetylated and deacetylated compounds are different, the textures observed for the deacetylated products, under crossed polarizer and analyzer were almost similar to those observed for acetylated products except for the compound, 1a. The compound 1a and deacetylated product show clearly distinct textures implying that they stabilize two different liquid crystal phases. The clearing points of deacetylated compounds are somewhat higher than those of the acetylated compounds, due to the fact that the degree of formation of hydrogen bonds increases with the deacetylation.

In the XRD pattern of deacetylated 1a compound, there was no peak in the small angle region and a sharp peak at $2 \theta, 10.93^{\circ}$ with a d-spacing of $d=8.09 \mathrm{~A}^{\circ}$ followed by a diffuse peak was observed. Whereas in the XRD pattern of deacetylated $1 \mathrm{~b}$, small peaks at $2 \theta, 7.68^{\circ}$ with $\mathrm{d}=11.50 \mathrm{~A}^{\circ}$ and at $2 \theta$ value $8.43^{\circ}$ with a d-spacing of $\mathrm{d}=10.48 \mathrm{~A}^{\circ}$ followed by a diffuse peak were

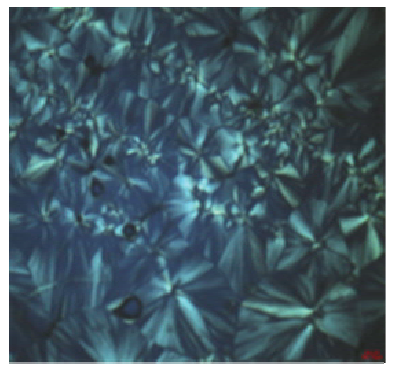

(a)

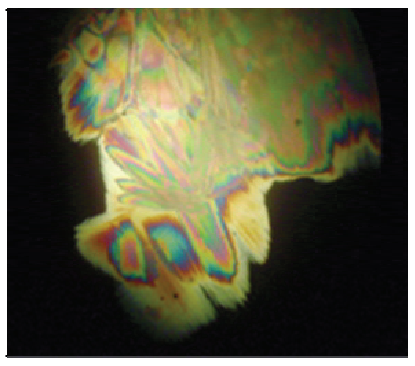

(b)

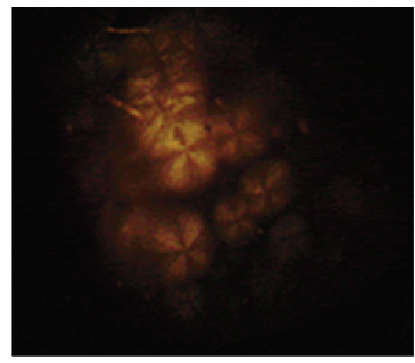

(c)

Figure 4: Optical textures of lyotropic mesophases of acetylated compounds (a) compound 1a, (b) compound $1 \mathrm{~b}$ and $(\mathrm{c})$ compound $1 \mathrm{c}$ in dichloromethane $(400 \times)$ taken under crossed polarizers

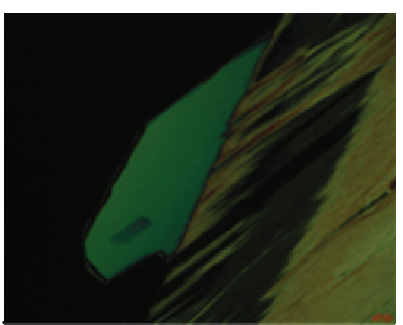

(a)

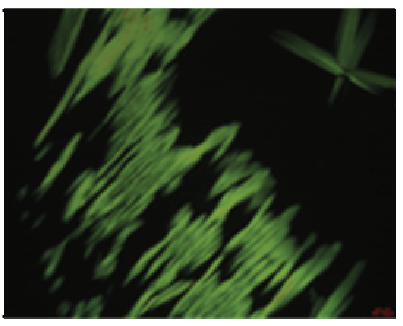

(d)

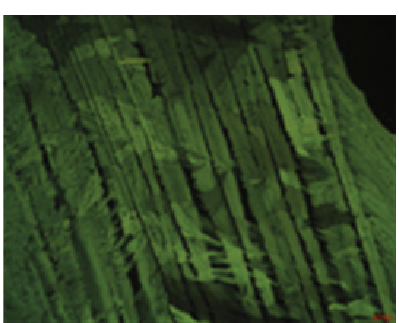

(b)

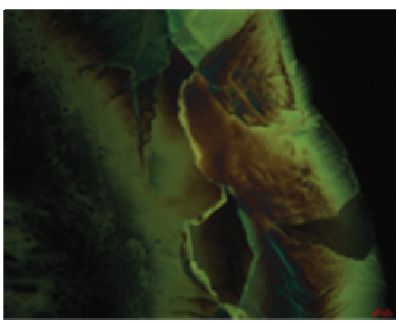

(e)

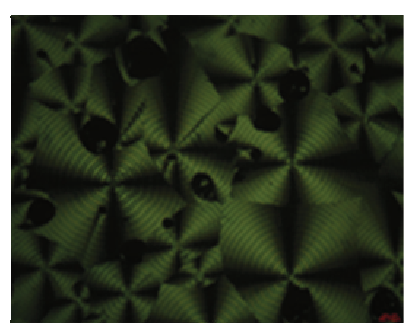

(c)

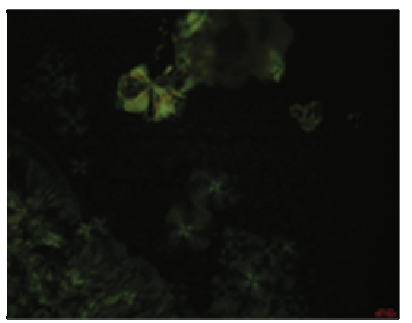

(f)

Figure 5: Thermotropic textures of deacetylated (a) compound 1a, (b) compound 1b, (c) compound 1c and lyotropic textures of deacetylated (d) compound 1a heated in $\mathrm{H}_{2} \mathrm{O}$, (e) compound $1 \mathrm{~b}$ in $\mathrm{CH}_{2} \mathrm{Cl}_{2}$ and (f) compound $1 \mathrm{c}$ in $\mathrm{CH}_{2} \mathrm{Cl}_{2}(400 \times)$ taken under crossed polarizers 
Table 3: Quantitative thermal analysis of deacetylated glucosides

\begin{tabular}{ccccc}
\hline $\begin{array}{c}\text { Deacetylated } \\
\text { compound }\end{array}$ & $\mathrm{m} . \mathrm{p}^{\mathrm{a}} /{ }^{\circ} \mathrm{C}$ & $\mathrm{c} \cdot \mathrm{p}^{\mathrm{b}} /{ }^{\circ} \mathrm{C}$ & $\begin{array}{c}2 \theta(\text { Small } \\
\text { angle region })\end{array}$ & $\begin{array}{c}\mathrm{d}-\text { Spacing/ } \\
\mathrm{A}^{\circ}\end{array}$ \\
\hline 1a & 85 & 110 & No peak & - \\
1b & 75 & 90 & 7.68 & 11.50 \\
$1 \mathrm{c}$ & 47 & 75 & 4.98 & 17.73 \\
\hline
\end{tabular}

${ }^{\mathrm{a}} \mathrm{m} . \mathrm{p}$; melting point, ${ }^{\mathrm{b}} \mathrm{c} . \mathrm{p}$; clearing point

observed suggesting a layered structure. One small peak at the $2 \theta$ value $4.98^{\circ}$ with a d-spacing of $17.73 \mathrm{~A}^{\circ}$ and a diffuse peak in the wide angle region were observed in the X-ray pattern of deacetylated 1c (Table 3). Hence, the deacetylated compounds are amphotropic liquid crystals. From these data we suggest that deacetylated compounds 1a and $1 \mathrm{~b}$ exhibit smectic B phase and compound 1c hexagonal columnar phase.

\section{CONCLUSION}

The three novel glucosides synthesized, $1 \mathrm{a}, 1 \mathrm{~b}$ and 1c clearly showed three different liquid crystalline mesophases, smectic A, smectic B and hexagonal columnar, respectively. The deacetylated compounds $1 \mathrm{a}$ and $1 \mathrm{~b}$ showed smectic B phases whereas compound 1c exhibited hexagonal columnar phase. All of these mesophases are observed upon cooling and hence these compounds show monotropic liquid crystal behaviour. They are amphotropic liquid crystals as well. Although the technical applications of carbohydrate liquid crystals have not yet been studied immensely like conventional liquid crystals, they can be widely exploited as surfactants, membrane builders and most importantly for drug delivery.

\section{Acknowledgement}

The authors thank the National Science Foundation (Grant RG/2007/FR/05), the National Research Council (Grant RG 06-25) and the University of Peradeniya (Grants $\mathrm{RG} / 2006 / 50 / \mathrm{S}, \mathrm{RG} / 2007 / \mathrm{C}-1 / 40 / \mathrm{S}$ and $\mathrm{RG} / 2008 / 48 / \mathrm{S}$ ) for research funding.

\section{REFERENCES}

1. Fodor-Csorba K., Jakli A., Vajda A., Prasad S. K., Rao D. S. S., Dong R. Y., Xu J. \& Galli G. (2006). Intercalated smectic A phases in banana-shaped liquid crystals with carbonate end groups. Chemphyschem 7(10): 2184-2188.

2. Jeffrey G. A. (1986). Carbohydrate liquid crystals. Accounts of Chemical Research 19: 170-171.

3. Mattsson S., Dahlstrom M. \& Karlsson S. (2007). A mild hydrolysis of ester medicated by lithium salts. Tetrahedron Letters 48: 2497-2499.

4. Smiths E., Engberts J. B. F. N., Kellogg R. M. \& van Doren H. A. (1996). Reliable method for the synthesis of aryl- $\beta$-D-glucopyranosides, using boron trifluoride-diethyl ether as catalyst. Journal of the Chemical Society, Perkins Transactions 1: 2873-2877.

5. Smiths E., Engberts J. B. F. N., Kellogg, R. M. \& van Doren H. A. (1997). Thermotropic and lyotropic liquid crystalline behavior of 4-alkoxyphenyl $\beta$-D-glucopyranosides. Liquid Crystals 23(4): 481-488.

6. Vill V. (1998). Carbohydrate Liquid Crystals. Available at $\mathrm{h}$ ttp://liqcryst.chemie.uni-hamburg.de/lcionline/article/ oldarticles/article_0698/articl e_2.htm, Accessed June 2008.

7. Vill V. \& Hashim R. (2002). Carbohydrate liquid crystals: structure-property relationship of thermotropic and lyotropic glycolipids. Current Opinion in Colloid and Interface Science 7: 395 - 409.

8. Wingert L. M., Jeffrey G. A., Cabaret D. \& Wakselman M. (1995). Liquid crystal properties of octyl 6'-Oalkylmelibiosides. Carbohydrate Research 275: 25 - 31 . 